\title{
Universiteit
}

Leiden

The Netherlands

\section{Robustness of edge states in graphene quantum dots}

\author{
Wimmer, M.; Akhmerov, A.R.; Guinea, F.
}

\section{Citation}

Wimmer, M., Akhmerov, A. R., \& Guinea, F. (2010). Robustness of edge states in graphene quantum dots. Physical Review B, 82, 045409. doi:10.1103/PhysRevB.82.045409

Version: $\quad$ Not Applicable (or Unknown)

License: $\quad$ Leiden University Non-exclusive license

Downloaded from: https://hdl.handle.net/1887/61285

Note: To cite this publication please use the final published version (if applicable). 


\title{
Robustness of edge states in graphene quantum dots
}

\author{
M. Wimmer and A. R. Akhmerov \\ Instituut-Lorentz, Universiteit Leiden, P.O. Box 9506, 2300 RA Leiden, The Netherlands \\ F. Guinea \\ Instituto de Ciencia de Materiales de Madrid, CSIC, Sor Juana Inés de la Cruz 3, E28049 Madrid, Spain
}

(Received 24 March 2010; revised manuscript received 17 June 2010; published 9 July 2010)

\begin{abstract}
We analyze the single-particle states at the edges of disordered graphene quantum dots. We show that generic graphene quantum dots support a number of edge states proportional to circumference of the dot over the lattice constant. Our analytical theory agrees well with numerical simulations. Perturbations breaking electron-hole symmetry such as next-nearest-neighbor hopping or edge impurities shift the edge states away from zero energy but do not change their total amount. We discuss the possibility of detecting the edge states in an antidot array and provide an upper bound on the magnetic moment of a graphene dot.
\end{abstract}

DOI: 10.1103/PhysRevB.82.045409

PACS number(s): 73.21.La, 73.22.Pr, 73.20.At, 73.22.-f

\section{INTRODUCTION}

The experimental discovery ${ }^{1,2}$ of graphene, a monolayer of carbon atoms, has opened room for new electronic devices (for reviews, see Refs. 3-5). A peculiarity of finite graphene sheets is the existence of electronic states localized at the boundary, so-called edge states.

A crystallographically clean zigzag edge was theoretically predicted to sustain zero-energy edge states. ${ }^{6-8}$ Later, it was shown ${ }^{9}$ that any generic graphene boundary not breaking electron-hole (e-h) subband (sublattice) symmetry also supports these zero-energy edge states. Similar states exist at zigzag edges of graphene bilayers ${ }^{10,11}$ and in other multilayered graphene systems. ${ }^{12}$ Experimentally, these states were observed in scanning tunneling microscope (STM) experiments near monatomic steps on a graphite surface. ${ }^{13-15}$

The presence of large number of localized states is important for the predicted edge magnetism in graphene nanoribbons, 7,16 a topic that has recently seen renewed interest in the context of graphene spintronics. ${ }^{17-21}$ Apart from edge magnetism, interacting edge states may also result in other correlated ground states. ${ }^{22-24}$

Edge states also play a role in confined geometries, when the edge to area ratio is large enough so that the electronic properties of the boundary may become dominant. One example for such a geometry is graphene quantum dots that have been under intense experimental study recently, ${ }^{25-30}$ with quantum-dot sizes in the range from a few tens of nanometer to micrometer. Another example is antidot arrays that have been subject of several theoretical studies ${ }^{31-34}$ and have also been realized experimentally. ${ }^{35-38}$

Different edges have been observed in graphite ${ }^{13-15,39}$ and graphene. ${ }^{40-43}$ In particular, the existence of boundaries with a long-range crystalline order in exfoliated graphene has been questioned. ${ }^{44}$ In addition, the existence of unsaturated dangling bonds at edges makes them reactive, and it is unclear how they are passivated. ${ }^{45-48}$ Hence, it is likely that graphene edges are perturbed and that the presence of edge distortions has to be taken into account.

The aim of our paper is to show that edge states can be expected in realistic disordered quantum dots. We also analyze the particular properties of edge states such as their number and compressibility. We start the analysis in Sec. II by using the theory of Ref. 9 for a relation between the number of edge states per unit length of a smooth boundary [see Fig. 1(b)] and the angle the boundary makes with respect to the crystallographic axis. We extend the earlier results by calculating the correction to the edge states number coming from the edge roughness [Fig. 1(c)]. Having the total number of edge states and their momentum distribution, we apply perturbation theory to see how confinement energy and particle-hole symmetry-breaking terms in the Hamiltonian shift the edge states from zero energy. Confinement energy spreads a delta functionlike peak in the density of states into a hyperbolic one. In contrast, particle-hole symmetrybreaking terms spread the edge states nearly homogeneously over a band of finite width. For realistic dot sizes around tens of nanometer, we find the latter to be more important.

In Sec. III, we perform numerical simulations on quantum dots of experimentally relevant sizes. These numerical calculations confirm our analytic results. We also study the magnetic field dependence of edge states in quantum dots. Whereas magnetic field spectroscopy of energy levels has up to now mainly been a useful tool to probe bulk states in graphene quantum dots, ${ }^{28,29,50}$ we show how to employ this technique also to identify edge states. In addition, we study the level statistics of edge states.

Finally in Sec. IV, we calculate an upper bound on the magnetic moment of a graphene dot due to edge-state polarization. We also give an upper bound on the relative weight of the edge states with respect to the bulk states. By knowing the magnitude of additional compressibility due to the edge states, we estimate parameters of an antidot lattice in which edge states would be visible in single-electron transistor (SET) experiments.

We conclude in Sec. V.

\section{ANALYTICAL CALCULATION OF THE EDGE-STATE DENSITY}

\section{A. Number of edge states}

The density of edge states per unit length was calculated for a smooth edge in Ref. 9, 


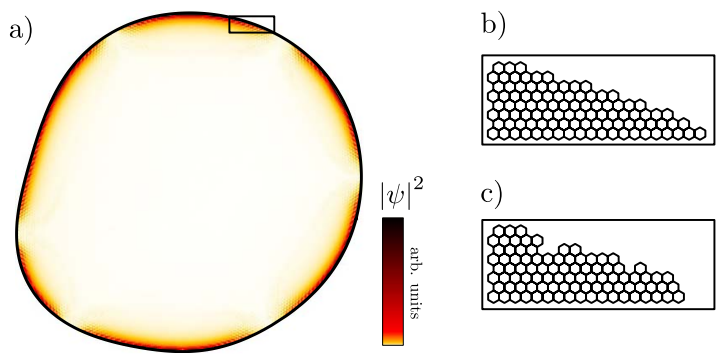

FIG. 1. (Color online) A graphene quantum dot. The excess density of states due to edge states is shown in a color plot (cf. footnote 49) as calculated for a quantum dot with a smooth boundary and no particle-hole symmetry-breaking perturbations (a). In general, edge states are present both near a smooth boundary (b) and a boundary with short-range disorder (c).

$$
\frac{d N}{d l}=\frac{2}{3 a}|\sin \phi|
$$

with $-\pi / 6<\phi<\pi / 6$ the angle boundary makes with a nearest armchair direction and $a$ the lattice constant. This expression is valid on the scales larger than the boundary roughness scale and another scale $\delta(\phi)$ dependent on boundary structure. For most boundary orientations, except the ones very close to armchair direction $\delta(\phi) \sim a$. Approximating the dot shape by a circle, and integrating Eq. (1) along the whole perimeter of the dot, we get

$$
N=\int_{0}^{L} \frac{d N}{d l} d L=\frac{4-2 \sqrt{3}}{\pi} \times \frac{L}{a}
$$

with $L$ circumference of the dot and $a$ the lattice constant. This density of states is the difference between total density of waves evanescent away from the boundary and the number of conditions the wave function must satisfy on the selected sublattice (see Ref. 9 for a more detailed description). If a small fraction $\alpha$ of random outermost atoms of the smooth edge oriented at angle $\phi$ with armchair direction is etched, the number of conditions for the wave function on the minority sublattice increases by

$$
\delta N=2 \alpha \sin \phi
$$

This leads to the reduction in the number of the edge states near an edge with atomic scale disorder,

$$
N^{\prime}=N(1-2 \alpha) \text {. }
$$

Note that Eq. (4) only gives the local density of low-energy edge states. It should not be confused with Lieb's theorem, ${ }^{51}$ which connects the number of states with exactly zero energy with the difference in the number of sublattice sites in a bipartite sublattice. Lieb's theorem was applied to graphene in Refs. 31, 33, and 52, and for a disordered quantum-dot geometry it predicts ${ }^{33}$ number of zero-energy modes $\sim \sqrt{L}$. Our analysis shows that there will be $\sim L$ low-energy edge states, although most of them do not lie at exactly zero energy. Hence, there is no contradiction with Lieb's theorem.

\section{B. Edge-state dispersion}

There are two different mechanisms which give finite energy to otherwise zero-energy edge states: the overlap between edge states on different sublattices, and terms breaking sublattice symmetry at the edge. The dispersion resulting from these perturbations can be calculated by applying degenerate perturbation theory, acting on the wave functions $\psi_{n}$, belonging exclusively to $A$ or $B$ sublattice. The longwavelength part of these wave functions is defined by the conformal invariance of Dirac equation so they can be approximated as plane waves belonging to one of the six facets of the dot with well-defined boundary condition, extended along the facet and decaying into the bulk. These wave functions have longitudinal momenta,

$$
k_{n} \sim \frac{n}{R}
$$

approximately equally spaced due to phase-space arguments.

We first estimate the energy dispersion due to edge-state overlap or in other words by finite-size effects. Particle-hole symmetry prevents coupling between states on the same sublattice so the dispersion of edge states in a finite system can be calculated from the matrix element between the edge states on different sublattices. These states are separated from each other by a distance of an order of the dot radius $R$ and their decay length away from the boundary is proportional to difference $k$ between their momentum and the momentum of the nearest Dirac point (Dirac momentum) so the energy is

$$
E(k) \sim \frac{v_{\mathrm{F}}}{R} e^{-k R},
$$

where $v_{\mathrm{F}}$ is the Fermi velocity and we set $\hbar=1$. We note that Eq. (6) is very similar to the energy of edge states in zigzag nanoribbons. ${ }^{53}$ Substituting the value of momentum of the edge states from Eq. (5) into Eq. (6), we calculate the density of edge states per unit energy,

$$
\rho(E) \equiv\left|\frac{d n}{d E}\right| \sim \frac{1}{E} .
$$

The atoms passivating the edge perturb the $\pi$ orbitals of carbon atoms to which they are bound. This interaction breaks the effective electron-hole symmetry of graphene around the Dirac point. Next-nearest-neighbor hopping is breaking this symmetry at the edges as well, ${ }^{54,55}$ and it was shown to be equivalent to the edge potential. ${ }^{56}$ The dispersion of the edge states near a zigzag edge due to these two perturbations is

$$
E=\left(\Delta \epsilon-t^{\prime}\right)[\cos (K)-1 / 2], \quad 2 \pi / 3<K<4 \pi / 3,
$$

where $K$ is the full momentum of the edge state, $\Delta \epsilon$ is the average strength of the edge potential, and $t^{\prime}$ the nextnearest-neighbor hopping strength. Despite, it is not straightforward to generalize this equation to an arbitrary orientation of the edge, the general effect of the electron-hole symmetrybreaking terms is to smear the zero-energy peak in the density of states into a band between energies of approximately 0 and $E_{0} \equiv \Delta \epsilon-t^{\prime}$ for the most localized states, while the 
more extended states are near the Dirac energy. The onedimensional Van Hove singularity in the density of states at $E=E_{0}$ will be smeared out, due to the presence of a minimum decay length of the edge states when the orientation of the boundary is not exactly zigzag. ${ }^{9}$

The energy due to finite size of the dot given by Eq. (6) is at best of an order of $E \sim v_{\mathrm{F}} / R \approx t a / R$. It is less than tens of millivolt for dots above $10 \mathrm{~nm}$ size. On the other hand, the energy due to the edge potentials and next-nearest-neighbor hopping [Eq. (8)] is likely to be around hundreds of millivolts. Accordingly in realistic dots with edge potentials and next-nearest-neighbor hopping term, edge states occupy the band between the Dirac point and $E_{0}$ with approximately constant density,

$$
\rho_{\text {edge }}=c(1-2 \alpha) \frac{R}{a\left|E_{0}\right|}
$$

with $c=8-4 \sqrt{3} \approx 1$.

\section{NUMERICAL RESULTS}

In order to confirm the analytical results of the previous sections, we have performed numerical simulations of the energy spectrum of graphene quantum dots with sizes relevant for experiments. In the following, we present results for a quantum dot with the shape of a deformed circle ${ }^{57}$ (cf. Fig. 1), characterized by an average radius $R$. Although we focus on a particular quantum dot here, we have found through numerical studies that the characteristic features of our results are independent from the details of the dot shape.

The numerical simulations are based on a tight-binding model of graphene with Hamiltonian,

$$
H=-\sum_{i, j} t_{i j} c_{i}^{\dagger} c_{j}+\text { H.c. }
$$

where the hopping $t_{i j}=t$ for nearest neighbors and $t_{i j}=t^{\prime}$ for next-nearest neighbors. ${ }^{5}$ The effects of a magnetic field are incorporated through the Peierls phase as ${ }^{58}$

$$
t_{i j} \rightarrow t_{i j} \times \exp \left[\frac{i e}{\hbar} \int_{\mathbf{x}_{i}}^{\mathbf{x}_{j}} d \mathbf{s} \mathbf{A}(\mathbf{x})\right],
$$

where $\mathbf{x}_{i}$ and $\mathbf{x}_{j}$ are the positions of atom $i$ and $j$, respectively, and $\mathbf{A}(\mathbf{x})$ is the magnetic vector potential.

The quantum dots are constructed by "cutting" the desired shape out of the hexagonal graphene grid. For a shape that is smooth on the length scale of the lattice constant as considered here, this results in edges with a locally well-defined orientation [smooth edges, see Fig. 1(b)]. In order to account for edge disorder on the lattice scale [rough edges, see Fig. 1(c)], we adopt the disorder model introduced in Ref. 59. Starting from the smooth edge, atoms at the boundary are removed randomly with probability $p$, with dangling bonds removed after each pass. This procedure is repeated $N_{\text {sweep }}$ times.

The energy spectrum of the dot tight-binding Hamiltonian is calculated numerically using standard direct eigenvalue algorithms $\mathrm{s}^{60}$ and matrix bandwidth reduction techniques ${ }^{61}$ if a large part of the spectrum is needed. In contrast, if only a
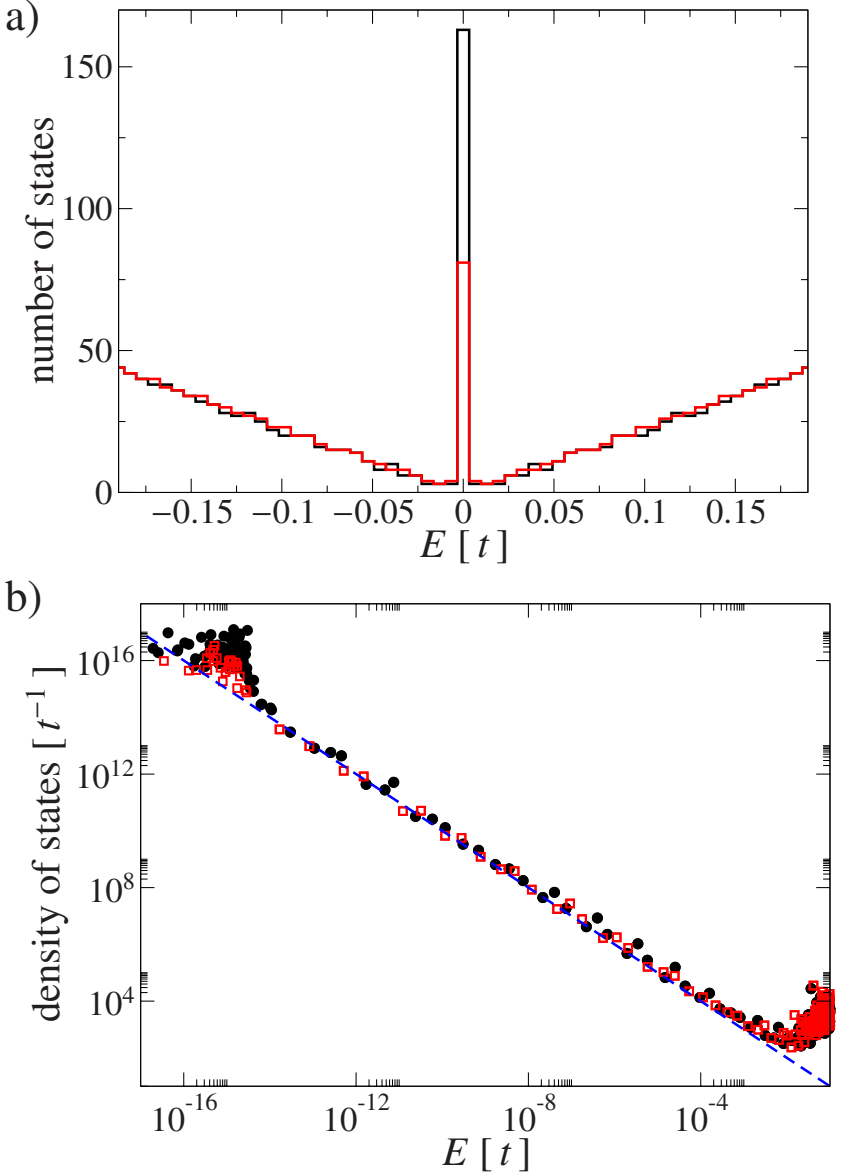

FIG. 2. (Color online) Electronic states in a graphene quantum dot close to the Dirac point. The graphene quantum dot has the shape of a deformed circle (see Fig. 1 and footnote 57) with $R$ $=160 a \approx 40 \mathrm{~nm}$, and we consider both smooth and rough edges as shown in Figs. 1(b) and 1(c), respectively. The parameters for the edge disorder are $N_{\text {sweep }}=5$ and $p=0.05$ (see the main text for a discussion of the edge disorder model). (a) Number of states per energy interval $\Delta E$ for a quantum dot with smooth (black lines) and rough edges [red lines (gray in print)], with $\Delta E=0.4 t / 61$. (b) Density of states estimated numerically from Eq. (12) for a quantum dot with smooth (black symbols) and rough edges [red symbols (gray in print)]. For comparison, the blue dashed line shows a $1 / E$ dependence.

few eigenvalues and eigenvectors are sought, we apply an iterative technique ${ }^{62}$ in shift-and-invert mode. ${ }^{63}$

\section{A. Systems with electron-hole symmetry}

We first focus on the electron-hole symmetric case, i.e., $t^{\prime}=0$ and the absence of potentials. Figure 2(a) shows the number of states $N(E)$ per energy interval $\Delta E$ for dots with smooth and rough edges. We can clearly identify the edge states close to $E=0$ and the linearly increasing bulk density of states. Approximating the circumference of the dot as $L$ $\approx 2 \pi R$, Eq. (2) predicts $N \approx 170$ edge states for a quantum dot with a smooth edge, which is in very good agreement with $N=169 \pm 6$ edge states obtained from the numerical simulation by summing over the three central bins, where the 
number of states differs noticeably from the linear bulk density of states. The number of edge states $N^{\prime}$ in the dot with atomic-scale disorder can be estimated from Eq. (3) by approximating $\alpha \approx p N_{\text {sweep }}$ yielding $N^{\prime} \approx 0.5 N$ for the disorder parameters used in the simulation $\left(N_{\text {sweep }}=5, p=0.05\right)$, again in good agreement with the numerical simulations.

In order to examine the behavior of the edge-state density of states in more detailed close to $E=0$, we estimate the density of states numerically as

$$
\rho\left(\left(E_{i+1}+E_{i}\right) / 2\right)=\frac{1}{E_{i+1}-E_{i}},
$$

where $E_{i}$ is the energy of the $i$ th state in the dot. Figure 2(b) shows the numerically computed $\rho(E)$ for quantum dots with smooth and rough edges. As predicted in Eq. (7), we find a $1 / E$ dependence close to $E=0$; quite remarkably, we find an excellent agreement with this scaling for more than ten orders of magnitude. The clustering of data points at $\rho(E)$ $=10^{16} t^{-1}$ is due to the finite precision in the numerical calculations. It should be noted that we found this remarkable agreement with theoretical predictions without averaging over an energy window or different dot shapes, implying that the spectrum of edge states is highly nonrandom even in a quantum dot with random shape. We come back to this point in Sec. III D.

\section{B. Broken electron-hole symmetry}

Next we focus on perturbations breaking the electron-hole symmetry. For this we consider a finite next-nearest-neighbor hopping $t^{\prime}$ uniformly within the quantum dot, as well as a random potential at the quantum-dot edge, where an energy $U_{0}$ is assigned to edge atoms with probability $p_{\text {edge }}$.

Figure 3(a) shows the number of states per energy window $\Delta E$ for finite $t^{\prime}$ but in the absence of an edge potential. In order to identify the edge states properly, we compare the numerical data including the edge states to the number of bulk states estimated from the linear Dirac density of states, ${ }^{5}$

$$
N_{\text {bulk }}(E)=\frac{2|E| R^{2}}{v_{\mathrm{F}}^{2}}
$$

approximating the area of the quantum dot as $A=\pi R^{2}$. The excess edge-state density of states can be clearly identified, both in the case of smooth and rough edges. The bulk density of states close to $E=0$ is unaffected by a finite $t^{\prime}$, the effect of electron-hole asymmetry on the bulk states only shows for energies $|E|>0.1 t$. The central edge-state peak observed for $t^{\prime}=0$ (cf. Fig. 2) is broadened and shifted toward the hole side but the total number of edge states remains unchanged from the $t^{\prime}=0$ case. The excess density due to the edge states is approximately constant in the energy range between $t^{\prime}=$ $-0.1 t$ and 0 , in accordance with the prediction from Eq. (9). As before, atomic scale edge disorder only results in a reduction in the total number of edge states.

The presence of an additional edge potential changes the energy range of the edge states. In Fig. 3(b), we show results for an average edge potential $\Delta \epsilon=0.05 t$. Correspondingly, the majority of the edge states occupies uniformly an energy

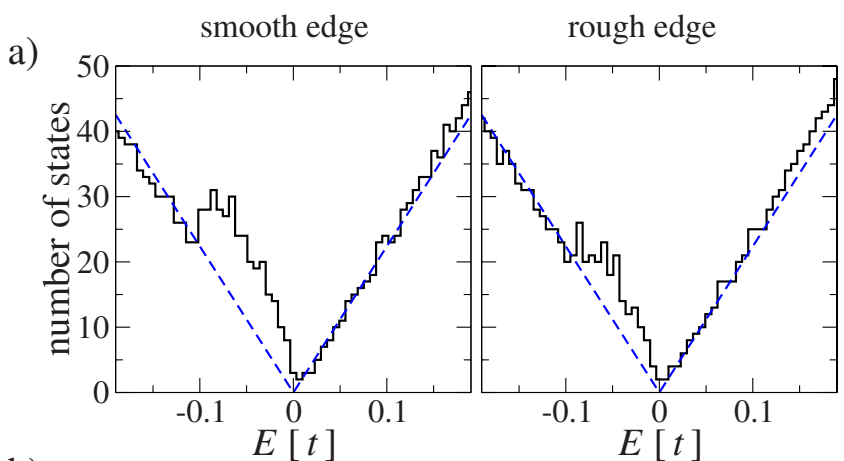

b)

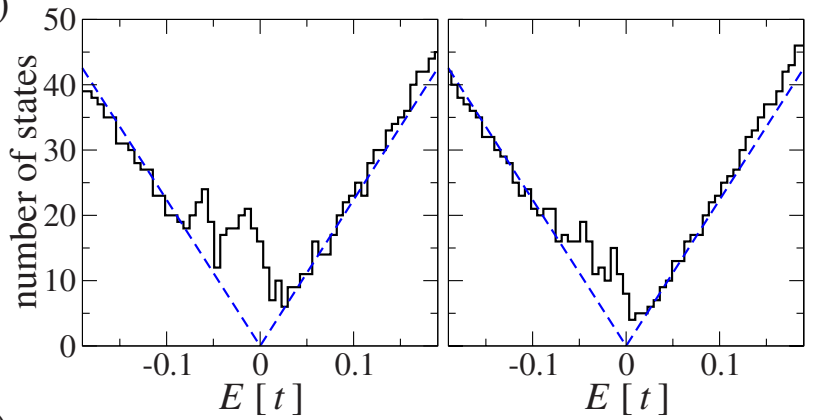

c)

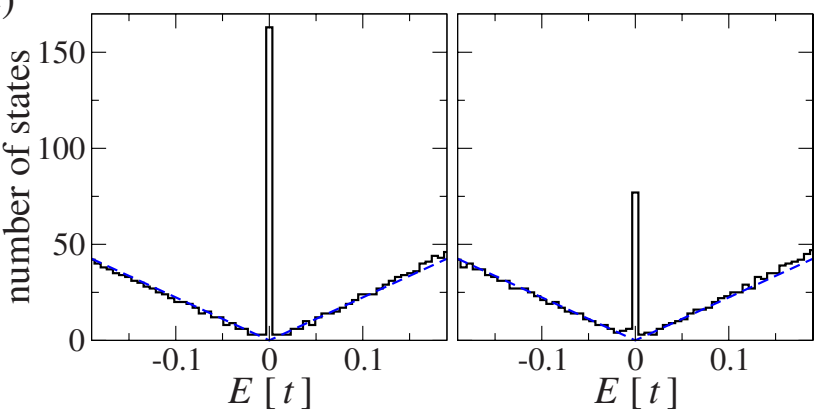

FIG. 3. (Color online) Number of states (black lines) per energy interval $\Delta E$ for a graphene quantum dot with smooth (left panels) and rough (right panels) edges. We show results for situations with broken electron-hole symmetry: (a) finite next-nearest-neighbor hopping and no edge potential $\left(t^{\prime}=0.1 t\right.$ and $\left.U_{0}=0\right)$ and (b) and (c) finite next-nearest-neighbor hopping including an edge potential $\left[t^{\prime}=0.1 t\right.$, with (b) $p_{\text {edge }}=0.25$ and $U_{0}=0.2 t$, and (c) $p_{\text {edge }}=1$ and $\left.U_{0}=0.1 t\right]$. The remaining parameters are as in Fig. 2. The blue dashed lines show the number of bulk states $N_{\text {bulk }}$ estimated from the linear density of states of the Dirac dispersion Eq. (13).

window between $\Delta \epsilon-t^{\prime}=-0.05 t$ and 0 . A few states can still be found beyond this energy window, as the randomness of the edge potential has been neglected in the arguments of Sec. II B. Instead, if the edge potential is uniform, the dispersion of the edge state due to next-nearest-neighbor hopping can be canceled exactly by $\Delta \epsilon=-t^{\prime}$, as shown in Fig. 3(c). This particular example strikingly shows the equivalence of next-nearest-neighbor hopping and an edge potential, as predicted in Ref. 56.

The narrowing of the energy bandwidth occupied by the edge state due to an edge potential may also be a possible explanation (among others ${ }^{64}$ ) for the fact that STM measurements on zigzag graphene edges found a peak in the density of states only a few tens of millielectron volt below the Dirac point, ${ }^{13,15}$ far less than expected from estimated values of the next-nearest-neighbor hopping. ${ }^{5}$ 


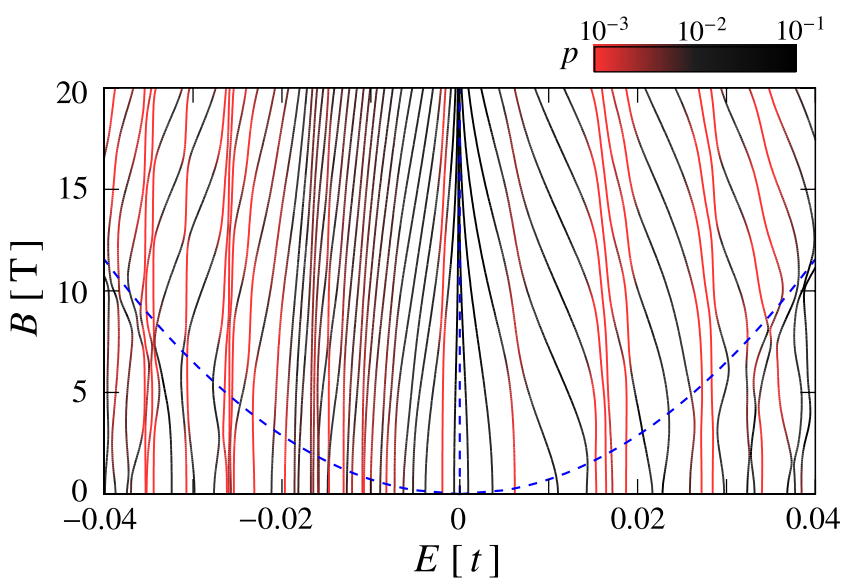

FIG. 4. (Color online) Magnetic field dependence of the energy levels (black lines) in a desymmetrized quantum dot with $R$ $=100 a$ (deformed circle as shown in Fig. 1, cf. footnote 57). The participation ratio $p$ of the states is color encoded, with the most strongly localized states in red. The blue dashed lines indicate the energy of the $n=0, \pm 1$ Landau levels of graphene. The calculations includes finite next-nearest-neighbor hopping $t^{\prime}=0.1 t$ and a random edge potential with $p_{\text {edge }}=0.25$ and $U_{0}=0.2 t$.

\section{Broken time-reversal symmetry: Finite magnetic field}

We now consider the effects of a finite magnetic field on the edge-state energies. The evolution of edge states in a magnetic field has been studied theoretically for special geometries and a particle-hole symmetric spectrum. ${ }^{65,66} \mathrm{Re}$ cently, the magnetic field dependence of the energy levels in a graphene quantum dot has been also been subject to an experimental investigation. ${ }^{28}$ However, in the theoretical calculations used to interpret these experiments the graphene edge states were excluded artificially. As we show below, the presence of edge states results in a much richer magnetic field dependence of energy levels in a graphene dot, in particular, when particle-hole symmetry is broken.

In Fig. 4, we show the numerically calculated magnetic field dependence of the energy levels in a graphene quantum dot close to the Dirac point, for finite $t^{\prime}$ and edge potential. In order to distinguish between edge and bulk states, we also plot the participation ratio, ${ }^{67,68}$

$$
p=\frac{\left(\sum_{i}|\psi(i)|^{2}\right)^{2}}{N \sum_{i}|\psi(i)|^{4}},
$$

where the index $i$ runs over atomic sites and $N$ denotes the total number of atoms in the dot. The participation ratio $p$ can be interpreted as the fraction of atoms occupied by an electron for a given energy level. Thus, $p \sim 1$ for extended states $(p \approx 0.3-0.4$ in quantum dots) and $p \ll 1$ for localized edge states $\left(p \approx 10^{-4}-10^{-2}\right)$.

Instead of a uniform flow of energy levels toward the $n$ $=$ Landau level as calculated in Ref. 28, we observe that the most strongly localized states only show a very weak magnetic field dependence (apart from avoided crossings), leading to a far richer energy spectrum. Note that this effect is most prominent on the hole side of the spectrum where the majority of the edge states reside, as can be simply seen by comparing the number of states for $E>0$ and $E<0$. This weak magnetic field dependence of the localized edge states can be understood from the fact that bulk states start to be affected by the magnetic field when the cyclotron radius becomes comparable to the dot size whereas edge-state energies are expected to only change significantly when the cyclotron radius becomes comparable to the edge-state decay length which is much smaller than the dot dimensions.

Note that this type of behavior is similar to the magnetic field dependence of the low-energy spectrum of graphene in the presence of lattice vacancies. ${ }^{69}$ In fact, such vacancies can be considered as internal edges and also carry a localized state.

Hence, magnetic field independent energy levels are characteristic for localized (edge) states. In the light of this observation, it would be very interesting to see if experiments can identify such states, which would be a strong indication for the presence of such states.

\section{Level statistics of edge states}

The bulk states of chaotic graphene quantum dots confined by lattice termination have been shown to follow the level statistics of the Gaussian orthogonal ensemble (GOE), as expected for a system with time-reversal symmetry ${ }^{70,71}$ (scattering at the quantum-dot boundary mixes the $K$ and $K^{\prime}$ valley). The edge states however are tied to the boundary of the quantum dot only, and should not necessarily follow the same level statistics as the extended states. Instead, being localized states they are rather expected to follow Poisson statistics, as has also been noted in Ref. 70 but not been demonstrated explicitly.

To check these expectations, we have studied the levelspacing distribution of edge states in quantum dots. For this purpose, we have identified edge states using the participation ratio and worked with the edge-state spectrum alone. This spectrum has been unfolded ${ }^{72}$ using the average density of states and scaled to an average level spacing of unity. The distribution $P(S)$ of the nearest-neighbor level spacings $S$ in the unfolded spectrum is then normalized such that $\int P(S) d S=1$ and $\int S P(S) d S=1$.

Figure 5 shows the level-spacing distributions for the electron-hole symmetric case $\left(t^{\prime}=0\right)$ and for broken electronhole symmetry $\left(t^{\prime}=0.1 t\right)$. Surprisingly, the edge states follow the GOE statistics if $t^{\prime}=0$. Only if a finite $t^{\prime}$ is included, they exhibit a statistics close to Poisson. These classifications are additionally corroborated by the integrated level-spacing distributions shown in the inset of Fig. 5.

This striking difference in level statistics can be explained by the different nature of the wave functions. The graphene Hamiltonian exhibits a chiral symmetry for $t^{\prime}=0$ that results in an equal occupation probability of sublattice A and B for every individual wave function. ${ }^{74}$ Since the edge wave function at a certain type of zigzag edge is nonzero only on one sublattice, every eigenstate for $t^{\prime}=0$ must also occupy another part of the boundary of the opposite kind, as illustrated in Fig. 6. This leads to an artificial long-range coupling between edge states and thus to level repulsion, resulting fi- 


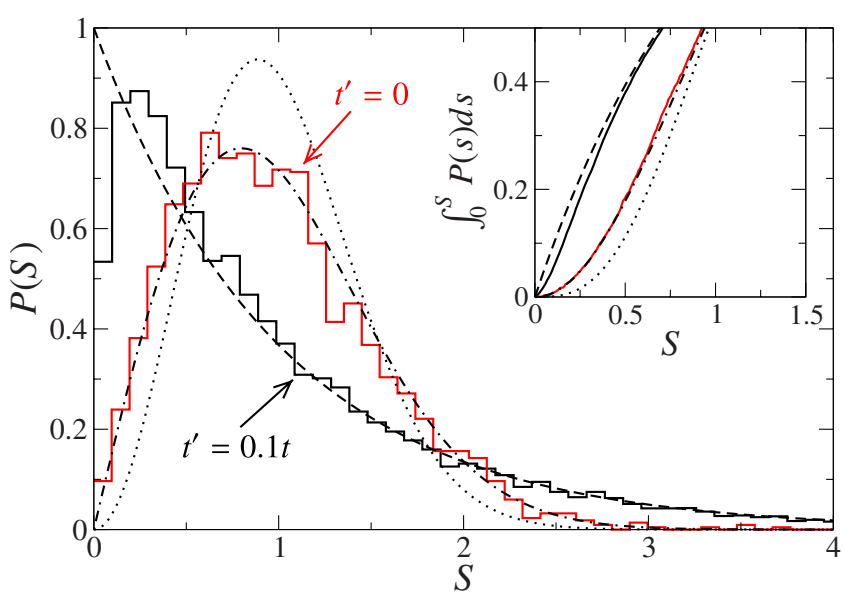

FIG. 5. (Color online) Level-spacing distributions for quantum dots with smooth edges for $t^{\prime}=0$ (solid red curve) and $t^{\prime}=0.1 t$ (solid black curve), together with the theoretical predictions for Poisson statistics (dashed line), the Gaussian orthogonal ensemble (dashed-dotted line), and the Gaussian unitary ensemble (dotted line). The inset shows details the integrated level spacing distribution for small level spacings $S$ (same line colors and types as the main plot). The level distribution statistics has been obtained by averaging individual level distributions from 100 quantum dots similar to the type given in footnote 57, with average radius $R$ $=160 \mathrm{a}$. A state has been identified as an edge state, if its participation ratio $p_{i}<0.05$ (Ref. 73). For $t^{\prime}=0$ we have also omitted all states with an energy smaller than the numerical precision.

nally in GOE statistics. If this chiral symmetry is broken, for example, by next-nearest-neighbor hopping, ${ }^{75}$ edge-state wave functions may be localized at a single edge only [Fig. 6(a)]. While edge states localized at the same part of the boundary still may feel level repulsion, parts that are further away may only interact via hybridization with bulk states which typically happens for edges states decaying further into the bulk, as seen in Fig. 6(b). For the type of quantum dots under consideration (Fig. 1), this results in six approximately independent series of energy levels, and hence an approximate Poisson statistics.

A finite next-nearest-neighbor hopping $t^{\prime}$ (or another chiral symmetry-breaking term) thus does not only change properties of the edge states quantitatively but leads to a striking, qualitatively different level statistics.

\section{DISCUSSION AND PHYSICAL IMPLICATIONS}

\section{A. Formation of magnetic moments at the edges}

An extensively discussed topic in the graphene literature is the formation of localized moments at boundaries. ${ }^{17,18,20,22-24,76,77}$ The previous analysis allows us to set approximate bounds on the maximum magnetic moment in a graphene quantum dot.

The interaction energy between two electrons of opposite spin in a boundary state of area $k_{i}^{-1} \times R \approx a \times R$ is

$$
E_{e e} \approx \frac{e^{2}}{R} \log \left(\frac{R}{a}\right),
$$

where $e$ is the electronic charge. States with energies $\mid \epsilon_{i}$ $-E_{\mathrm{F}} \mid \leq E_{e e}^{i}$ will be spin polarized. Since the density of edge

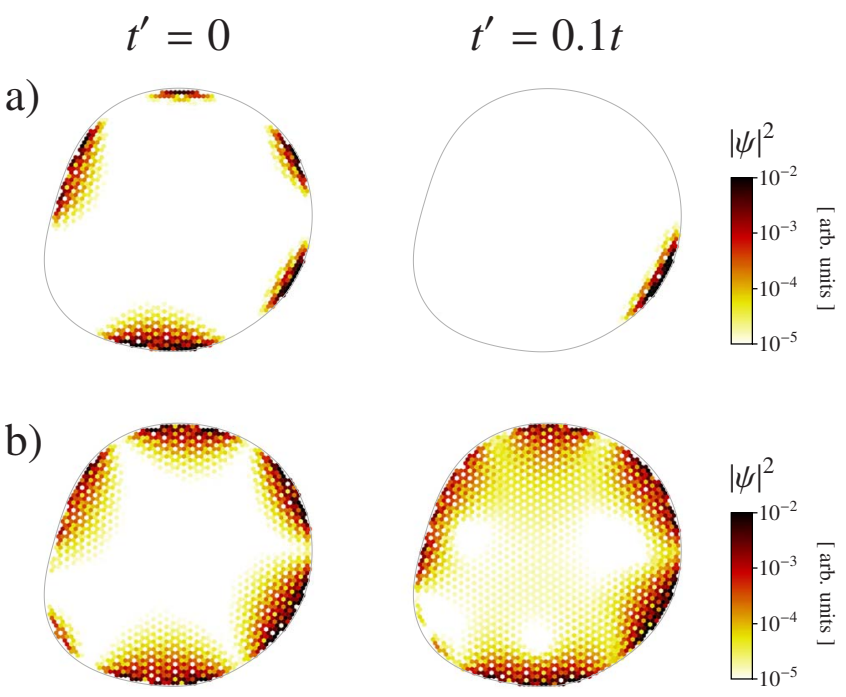

FIG. 6. (Color online) Color plot (cf. footnote 49) of wavefunction density in a graphene quantum dot (shape as described in footnote 57) for the electron-hole (e-h) symmetric case $\left(t^{\prime}=0\right.$, left column) and for broken e-h symmetry $\left(t^{\prime}=0.1 t\right.$, right column $)$ on the examples of a mode that is (a) strongly decaying and (b) slowly decaying into the bulk. Note that for presentation purposes we have chosen a rather small dot $(R=30 a)$ but the behavior does not change qualitatively for larger dots.

states is nearly constant and given by Eq. (9), the position of the Fermi level is not relevant. Using the density of states given in Eq. (9), we obtain for the number of spins in a quantum dot,

$$
\begin{aligned}
N_{\text {spins }} & \approx E_{e e} \rho_{\text {edge }} \\
& =c(1-2 \alpha) \frac{e^{2}}{a E_{0}} \log \left(\frac{R}{a}\right) \\
& \sim 20(1-2 \alpha) \log \left(\frac{R}{a}\right),
\end{aligned}
$$

where for last estimate we took $E_{0}=0.3 \mathrm{eV}$. The maximal number of polarized spins depends only logarithmically on the size of the dot.

In general, the states at the edge of a quantum dot will belong to one of the two sublattices with equal probability. States localized at different sublattices interact antiferromagnetically. ${ }^{78}$ If we neglect this interaction, we expect a maximum magnetic moment comparable with $N_{\text {spins. }}$. When the antiferromagnetic interaction contributes to the formation of the total magnetic moment, its value will be proportional to the number of uncompensated sites at the edges, which will scale as $\sqrt{N_{\text {spins }}}$.

\section{B. Fraction of edge states}

Our results suggest that edge and bulk states can coexist in a range of energy of order $E_{0}$ near the Dirac point. From Eqs. (9) and (13), the average ratio between edge and bulk states in this energy range is 


$$
\frac{N_{\text {edge }}}{N_{\text {bulk }}} \approx c(1-2 \alpha) \frac{v_{\mathrm{F}}^{2}}{E_{0}^{2} a R} .
$$

This gives for a diameter of $100 \mathrm{~nm}$ and $E_{0}=0.3 \mathrm{eV}$ an upper bound of $N_{\text {edge }} / N_{\text {bulk }} \lesssim 1 / 2$.

\section{Detection in antidot lattices}

A conclusive way of detecting the existence of edge states can be the measurement of their contribution to the electronic compressibility. It is hard to detect the edge states in a single quantum dot because the ground-state properties are dominated by the charging energy. Also, the contribution of edge states to the density of states in most large-scale samples will be negligible compared to the bulk contribution. However it is possible to circumvent both problems in antidot lattices. The Coulomb energy does not play a role in this case due to absence of confinement. On the other hand, the existence of multiple antidots allows us to reach a large edge-area ratio. To estimate whether it is possible to detect edge states, we use the value of minimal compressibility (or the minimal density of states) of bulk graphene Ref. 79,

$$
\frac{\partial \mu}{\partial n}=3 \times 10^{-10} \mathrm{meV} \mathrm{cm}^{2}
$$

and we assume that the width of the band of edge states is around $E_{0} \approx 0.3 \mathrm{eV}$.

We consider an antidot lattice with antidot size $L$ of the same order of magnitude as the antidot spacing. Using the analysis in the previous section, the density of states per unit area associated to the edge states is

$$
N_{\text {area }}^{-1}(E) \approx E_{0} a L \text {. }
$$

Comparing this expression with Eq. (18), and using $E_{0}$ $\approx 0.3 \mathrm{eV}$, we find that the contribution from the edge states is comparable to the bulk inverse compressibility for $L$ $\lesssim 1 \mu \mathrm{m}$. Hence, the additional density of states near the edge will be visible in compressibility measurements using a single-electron transistor (SET) since the size of the SET tip is around $100 \mathrm{~nm} .{ }^{79}$ Our results may be the reason of $p$ doping observed in antidot lattices experimentally. ${ }^{80,81}$

\section{CONCLUSIONS}

We have analyzed generic properties of the electronic spectrum in graphene quantum dots. We find that some of the electronic states will be localized at the edges and form a narrow band. The density of states in this band is $\propto 1 / E$ in graphene dot without electron-hole symmetry-breaking perturbations. In presence of such perturbations, the density of the edge states is approximately constant and scales as $R / a E_{0}$, where $R$ is the dot radius, $a$ is the lattice constant, and $E_{0}$ is an energy scale which describes the edge potentials and next-nearest-neighbor hopping.

If chiral symmetry is present, the edge states experience strong level repulsion and are described by the Gaussian orthogonal ensemble. Chiral symmetry-breaking terms (such as next-nearest-neighbor hopping) however lift this spurious level repulsion leading to the Poissonian statistics expected for localized states. In contrast, extended states will be described by the orthogonal or unitary ensembles, depending on the strength of the intervalley scattering at the boundaries. $^{70,82}$

Having an analytical model for the edge states allows us to estimate the maximum spin polarization due to the presence of edge states. We predict that the additional density of states due to edge states will be visible in SET experiments. Effect of edge states on transport in quantum dots and more detailed investigation of interaction effects remains a direction for further research.

\section{ACKNOWLEDGMENTS}

We are grateful for useful discussions to C. W. J. Beenakker, K. Ensslin, A. K. Geim, I. V. Grigorieva, K. S. Novoselov, J. H. Smet, and C. Stampfer. F.G. is supported by MEC (Spain) under Grants No. FIS2008-00124 and CONSOLIDER No. CSD2007-00010, and also the Comunidad de Madrid, through CITECNOMIK. M.W. is supported by the Deutscher Akademischer Austausch Dienst DAAD. A.A. is supported by the Dutch Science Foundation NWO/FOM and by the Eurocores program EuroGraphene.
${ }^{1}$ K. S. Novoselov, A. K. Geim, S. V. Morozov, D. Jiang, Y. Zhang, S. V. Dubonos, I. V. Grigorieva, and A. A. Firsov, Science 306, 666 (2004).

${ }^{2}$ K. S. Novoselov, D. Jiang, F. Schedin, T. J. Booth, V. V. Khotkevich, S. V. Morozov, and A. K. Geim, Proc. Natl. Acad. Sci. U.S.A. 102, 10451 (2005).

${ }^{3}$ A. K. Geim and K. S. Novoselov, Nature Mater. 6, 183 (2007).

${ }^{4}$ P. Avouris, Z. Chen, and V. Perebeinos, Nat. Nanotechnol. 2, 605 (2007).

${ }^{5}$ A. H. Castro Neto, F. Guinea, N. M. R. Peres, K. S. Novoselov, and A. K. Geim, Rev. Mod. Phys. 81, 109 (2009).

${ }^{6}$ D. J. Klein, Chem. Phys. Lett. 217, 261 (1994).

${ }^{7}$ M. Fujita, K. Wakabayashi, K. Nakada, and K. Kusakabe, J. Phys. Soc. Jpn. 65, 1920 (1996).
${ }^{8}$ K. Nakada, M. Fujita, G. Dresselhaus, and M. S. Dresselhaus, Phys. Rev. B 54, 17954 (1996).

${ }^{9}$ A. R. Akhmerov and C. W. J. Beenakker, Phys. Rev. B 77, 085423 (2008).

${ }^{10}$ E. V. Castro, N. M. R. Peres, J. M. B. Lopes dos Santos, A. H. Castro Neto, and F. Guinea, Phys. Rev. Lett. 100, 026802 (2008).

${ }^{11}$ B. Sahu, H. Min, A. H. MacDonald, and S. K. Banerjee, Phys. Rev. B 78, 045404 (2008).

${ }^{12}$ E. V. Castro, N. M. R. Peres, and J. M. B. Lopes dos Santos, EPL 84, 17001 (2008).

${ }^{13}$ Y. Niimi, T. Matsui, H. Kambara, K. Tagami, M. Tsukada, and H. Fukuyama, Appl. Surf. Sci. 241, 43 (2005).

${ }^{14}$ Y. Kobayashi, K.-I. Fukui, T. Enoki, K. Kusakabe, and Y. 
Kaburagi, Phys. Rev. B 71, 193406 (2005).

${ }^{15}$ Y. Niimi, T. Matsui, H. Kambara, K. Tagami, M. Tsukada, and H. Fukuyama, Phys. Rev. B 73, 085421 (2006).

${ }^{16}$ K. Nakada, M. Igami, and M. Fujita, J. Phys. Soc. Jpn. 67, 2388 (1998).

${ }^{17}$ Y.-W. Son, M. L. Cohen, and S. G. Louie, Nature (London) 444, 347 (2006)

${ }^{18}$ J. Fernández-Rossier and J. J. Palacios, Phys. Rev. Lett. 99, 177204 (2007).

${ }^{19}$ M. Ezawa, Phys. Rev. B 76, 245415 (2007).

${ }^{20}$ O. V. Yazyev and M. I. Katsnelson, Phys. Rev. Lett. 100, 047209 (2008).

${ }^{21}$ M. Wimmer, I. Adagideli, S. Berber, D. Tománek, and K. Richter, Phys. Rev. Lett. 100, 177207 (2008).

${ }^{22}$ B. Wunsch, T. Stauber, and F. Guinea, Phys. Rev. B 77, 035316 (2008).

${ }^{23}$ B. Wunsch, T. Stauber, F. Sols, and F. Guinea, Phys. Rev. Lett. 101, 036803 (2008).

${ }^{24}$ I. Romanovsky, C. Yannouleas, and U. Landman, Phys. Rev. B 79, 075311 (2009).

${ }^{25}$ L. A. Ponomarenko, F. Schedin, M. I. Katsnelson, R. Yang, E. W. Hill, K. S. Novoselov, and A. K. Geim, Science 320, 356 (2008).

${ }^{26}$ J. Güttinger, C. Stampfer, S. Hellmüller, F. Molitor, T. Ihn, and K. Ensslin, Appl. Phys. Lett. 93, 212102 (2008).

${ }^{27}$ C. Stampfer, J. Güttinger, F. Molitor, D. Graf, T. Ihn, and K. Ensslin, Appl. Phys. Lett. 92, 012102 (2008).

${ }^{28}$ J. Güttinger, C. Stampfer, F. Libisch, T. Frey, J. Burgdörfer, T. Ihn, and K. Ensslin, Phys. Rev. Lett. 103, 046810 (2009).

${ }^{29}$ S. Schnez, F. Molitor, C. Stampfer, J. Guettinger, I. Shorubalko, T. Ihn, and K. Ensslin, Appl. Phys. Lett. 94, 012107 (2009).

${ }^{30}$ J. Güttinger, T. Frey, C. Stampfer, T. Ihn, and K. Ensslin, arXiv:1002.3771 (unpublished).

${ }^{31}$ N. Shima and H. Aoki, Phys. Rev. Lett. 71, 4389 (1993).

${ }^{32}$ T. G. Pedersen, C. Flindt, J. Pedersen, N. A. Mortensen, A.-P. Jauho, and K. Pedersen, Phys. Rev. Lett. 100, 136804 (2008).

${ }^{33}$ M. Vanević, V. M. Stojanović, and M. Kindermann, Phys. Rev. B 80, 045410 (2009).

${ }^{34}$ J. A. Fürst, J. G. Pedersen, C. Flindt, N. A. Mortensen, M. Brandbyge, T. G. Pedersen, and A.-P. Jauho, New J. Phys. 11, 095020 (2009).

${ }^{35}$ T. Shen, Y. Q. Wu, M. A. Capano, L. P. Rokhinson, L. W. Engel, and P. D. Ye, Appl. Phys. Lett. 93, 122102 (2008).

${ }^{36}$ J. Eroms and D. Weiss, New J. Phys. 11, 095021 (2009).

${ }^{37}$ J. Bai, X. Zhong, S. Jiang, Y. Huang, and X. Duan, Nat. Nanotechnol. 5, 190 (2010).

${ }^{38}$ R. Balog, B. Jørgensen, L. Nilsson, M. Andersen, E. Rienks, M. Bianchi, M. Fanetti, E. Lægsgaard, A. Baraldi, S. Lizzit, Z. Sljivancanin, F. Besenbacher, B. Hammer, T. G. Pedersen, P. Hofmann, and L. Hornekær, Nature Mater. 9, 315 (2010).

${ }^{39}$ L. G. Cançado, M. A. Pimenta, B. R. A. Neves, M. S. S. Dantas, and A. Jorio, Phys. Rev. Lett. 93, 247401 (2004).

${ }^{40}$ A. L. Vázquez de Parga, F. Calleja, B. Borca, M. C. G. Passeggi, Jr., J. J. Hinarejos, F. Guinea, and R. Miranda, Phys. Rev. Lett. 100, 056807 (2008).

${ }^{41}$ X. Jia, M. Hofmann, V. Meunier, B. G. Sumpter, J. CamposDelgado, J.-M. Romo-Herrera, H. Son, Y.-P. Hsieh, A. Reina, J. Kong, M. Terrones, and M. S. Dresselhaus, Science 323, 1701 (2009).

${ }^{42}$ C. Ö. Girit, J. C. Meyer, R. Erni, M. D. Rossell, C. Kisielowski,
L. Yang, C.-H. C.-H. Park, M. F. Crommie, M. L. Cohen, S. G. Louie, and A. Zettl, Science 323, 1705 (2009).

${ }^{43}$ Z. Liu, K. Suenaga, P. J. F. Harris, and S. Iijima, Phys. Rev. Lett. 102, 015501 (2009).

${ }^{44}$ C. Casiraghi, A. Hartschuh, H. Qian, S. Piscanec, C. Georgi, A. Fasoli, K. S. Novoselov, D. M. Basko, and A. C. Ferrari, Nano Lett. 9, 1433 (2009).

${ }^{45}$ Y. J. Xu and J. Q. Li, Chem. Phys. Lett. 400, 406 (2004).

${ }^{46}$ D. E. Jiang, B. G. Sumpter, and S. Dai, J. Phys. Chem. B 110, 23628 (2006).

${ }^{47}$ F. Cervantes-Sodi, G. Csányi, S. Piscanec, and A. C. Ferrari, Phys. Rev. B 77, 165427 (2008).

${ }^{48}$ B. Huang, F. Liu, J. Wu, B.-L. Gu, and W. Duan, Phys. Rev. B 77, 153411 (2008).

${ }^{49}$ Close to a boundary, the edge states occupy a single sublattice only. In order to avoid the oscillatory pattern on the lattice scale that inevitably arises when plotting both sublattices simultaneously, for every unit cell we only plot the atom with the largest occupation probability.

${ }^{50}$ S. Schnez, K. Ensslin, M. Sigrist, and T. Ihn, Phys. Rev. B 78, 195427 (2008).

${ }^{51}$ E. H. Lieb, Phys. Rev. Lett. 62, 1201 (1989).

${ }^{52}$ M. Ezawa, Physica E 42, 703 (2010).

${ }^{53}$ A. R. Akhmerov, J. H. Bardarson, A. Rycerz, and C. W. J. Beenakker, Phys. Rev. B 77, 205416 (2008).

${ }^{54}$ N. M. R. Peres, F. Guinea, and A. H. Castro Neto, Phys. Rev. B 73, 125411 (2006).

${ }^{55}$ K. Sasaki, S. Murakami, and R. Saito, Appl. Phys. Lett. 88, 113110 (2006).

${ }^{56}$ K.-i. Sasaki, Y. Shimomura, Y. Takane, and K. Wakabayashi, Phys. Rev. Lett. 102, 146806 (2009).

${ }^{57}$ The dot has the shape of a deformed disk, with a radius $R(\theta)$ depending on the angle of direction $\theta$. The numerical calculations presented in the text use $R(\theta)=R+0.2 R \sin (\theta)$ $+0.05 R \sin (2 \theta)-0.025 R \sin (3 \theta)+0.02 R \sin (4 \theta)-0.01 R \sin (5 \theta)$.

${ }^{58}$ R. Peierls, Z. Phys. 80, 763 (1933).

${ }^{59}$ E. R. Mucciolo, A. H. Castro Neto, and C. H. Lewenkopf, Phys. Rev. B 79, 075407 (2009).

${ }^{60}$ E. Anderson, Z. Bai, C. Bischof, S. Blackford, J. Demmel, J. Dongarra, J. Du Croz, A. Greenbaum, S. Hammarling, A. McKenney, and D. Sorensen, LAPACK Users' Guide, 3rd ed. (Society for Industrial and Applied Mathematics, Philadelphia, PA, 1999).

${ }^{61}$ N. E. Gibbs, W. G. Poole,Jr., and P. K. Stockmeyer, SIAM J. Numer. Anal. 13, 236 (1976).

${ }^{62}$ R. B. Lehoucq, D. C. Sorensen, and C. Yang, ARPACK Users' Guide: Solution of Large-Scale Eigenvalue Problems with Implicitly Restarted Arnoldi Methods (Society for Industrial and Applied Mathematics, Philadelphia, PA, 1998).

${ }^{63}$ For the solution of the sparse linear system arising in the shiftand-invert problem, we apply the MUMPS package: P. R. Amestoy, I. S. Duff, J. Koster, and J.-Y. L'Excellent, SIAM J. Matrix Anal. Appl. 23, 15 (2001).

${ }^{64}$ Ken-ichi Sasaki, Kentaro Sato, Riichiro Saito, Jie Jiang, Seiichiro Onari, and Yukio Tanaka, Phys. Rev. B 75, 235430 (2007).

${ }^{65}$ D. A. Bahamon, A. L. C. Pereira, and P. A. Schulz, Phys. Rev. B 79, 125414 (2009).

${ }^{66}$ S. C. Kim, P. S. Park, and S.-R. Eric Yang, Phys. Rev. B 81, 085432 (2010) 
${ }^{67}$ R. J. Bell and P. Dean, Discuss. Faraday Soc. 50, 55 (1970).

${ }^{68}$ R. J. Bell, Rep. Prog. Phys. 35, 1315 (1972).

${ }^{69}$ F. Libisch, S. Rotter, J. Güttinger, C. Stampfer, and J. Burgdörfer, Phys. Rev. B 81, 245411 (2010).

${ }^{70}$ J. Wurm, A. Rycerz, I. Adagideli, M. Wimmer, K. Richter, and H. U. Baranger, Phys. Rev. Lett. 102, 056806 (2009).

${ }^{71}$ F. Libisch, C. Stampfer, and J. Burgdörfer, Phys. Rev. B 79, 115423 (2009).

${ }^{72}$ M. L. Mehta, Random Matrices (Elsevier/Academic Press, Amsterdam, 2004).

${ }^{73}$ The result does not change qualitatively if the threshold participation ratio is changed. In particular, the classification into the different types of level statistics does not depend on this threshold.
${ }^{74}$ L. Brey and H. A. Fertig, Phys. Rev. B 73, 235411 (2006).

${ }^{75}$ In Ref. 70 , the chiral symmetry was broken by a mass term.

${ }^{76}$ K. Harigaya, Chem. Phys. Lett. 340, 123 (2001).

${ }^{77}$ K. Harigaya and T. Enoki, Chem. Phys. Lett. 351, 128 (2002).

${ }^{78}$ L. Brey, H. A. Fertig, and S. Das Sarma, Phys. Rev. Lett. 99, 116802 (2007).

${ }^{79}$ J. Martin, N. Akerman, G. Ulbricht, T. Lohmann, J. H. Smet, K. von Klitzing, and A. Yacoby, Nat. Phys. 4, 144 (2008).

${ }^{80}$ E. H. Lee, K. Balasubramanian, R. T. Weitz, M. Burghard, and K. Kern, Nat. Nanotechnol. 3, 486 (2008).

${ }^{81}$ S. Heydrich, M. Hirmer, C. Preis, T. Korn, J. Eroms, D. Weiss, and C. Schüller, arXiv:1006.2067 (unpublished).

${ }^{82}$ H. D. Raedt and M. I. Katsnelson, JETP Lett. 88, 607 (2009). 